\title{
Discrete Variable Quantum Key Distribution in Millimeter-Wave and $\mathrm{THz}$ Regions
}

This paper was downloaded from TechRxiv (https://www.techrxiv.org).

\section{LICENSE}

CC BY 4.0

SUBMISSION DATE / POSTED DATE

02-08-2021 / 09-08-2021

\section{CITATION}

Ramos, Rubens (2021): Discrete Variable Quantum Key Distribution in Millimeter-Wave and THz Regions. TechRxiv. Preprint. https://doi.org/10.36227/techrxiv.15091338.v1

DOI

10.36227/techrxiv.15091338.v1 


\title{
Discrete Variable Quantum Key Distribution in Millimeter-Wave and $\mathrm{THz}$ Regions
}

\author{
V. F. Guedes, F. A. Mendonca, J. B. R. Silva, R. V. Ramos
}

\begin{abstract}
Quantum key distribution allows secure communication between spatially distant users. It has been successfully implemented in the infrared portion of the spectrum in both, fiber-based and free space optical channels. A natural step to be followed is to realize QKD in other portions of the electromagnetic spectrum, like microwave and millimeter-wave regions. In this direction, the present work discusses the implementation of the B92 QKD protocol in millimeter-wave using glow discharge device-based single-photon detector.
\end{abstract}

Index Terms - Single-photon detector, quantum key distribution, millimeter-wave, THz, glow discharge detectors.

\section{INTRODUCTION}

Q uantum key distribution (QKD) was the first commercially available quantum technology, implemented using the infrared portion of the electromagnetic spectrum, that allows secure communication between spatially distant users [1-5]. It has been successfully implemented in fiber-based and freespace optical networks. The implementation of QKD in the infrared region was possible because single-photon detectors (SPDs) in this region are easily built with silicon and InGaAs avalanche photodiodes (APD) or superconductors [6-10]. On the other hand, the $5 \mathrm{G}$ and $6 \mathrm{G}$ technologies are promising a plethora of new services [11] that will impact the industry, economy and peoples' lives. Nevertheless, quantum services are not included in this package. In this direction, it is natural to consider the realization of quantum protocols, like QKD, using microwave $(30-300 \mathrm{GHz})$ or millimetric wave (MMW) (0.3$10 \mathrm{THz}$ ) in order to provide a very high security level for those services. The firsts attempt in this direction used continuous variable QKD protocol, thermal light states and homodyne detection [12-14]. Up to now they have not been implemented. Here, we have a different proposal, we discuss how to implement the discrete variable B92 QKD protocol using SPDs that work in the MMW region. This SPD uses a glow discharge device (GDD), a miniature neon indicator lamp, operating in the up-conversion mode. Thus, differently of earlier proposals, our scheme uses discrete variable, coherent states and direct detection. Therefore, our scheme is cheap and it can be implemented with current technology.

The present work is outlined as follows: in Section II the SPD in the MMW portion of the spectrum is reviewed; in Section III the QKD protocol is explained and the conclusions are drawn in Section IV.

\section{Glow Discharge Device-Based Single-Photon DETECTOR IN THE MILLIMETER-WAVE AND THZ REGIONS}

Miniature neon indicator lamps are GDDs that have been used in direct and heterodyne detection of microwave and MMW [15-22]. The detection by the GDD can be inferred from two ways: I) Slight variation of the GDD electrical current. II) Slight variation of the power of the light emitted by the lamp. In the first case, although the internal gain of the GDD (due to the cascade of ionizations) seems to be very high, the power of a single-photon in the MMW region is very low, making the electrical current variation very hard to be detected in practice. In the second case, the GDD works as an up-converter device, absorbing MMW or THz photons and emitting visual light [20]. The last is the operation mode used in the GDD-based SPD. Electrons accelerated by the electrical field between the lamp terminals increase their kinetic energy slightly by absorbing $\mathrm{MMW} / \mathrm{THz}$ radiation, allowing them to participate in the ionization and excitation processes by colliding with atoms. When the atom is excited after a lifetime it releases the energy emitting a photon in the visual light wavelength. The photon emitted is detected by silicon avalanche photodiode (Si-SPD). Hence, the single-photon detector here considered is composed by a GDD working in the up-converter mode and a silicon APD-based SPD [23]. The scheme is shown in Fig. 1.

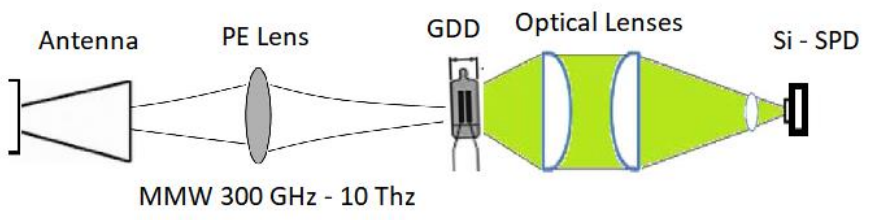

Fig. 1. Detection of MMW single-photon using a miniature neon lamp working in the up-converter mode and a silicon APD-based SPD detector.

A discharge can be fired when the voltage applied to GDD is larger than the breakdown voltage, $V_{B}$. Hence, the GDD is operated in the Geiger mode: the GDD is fed by a DC power supply $V$ in series with a signal generator the produces a narrow electrical pulse with peak voltage $V_{G}$ only when a MMW photon is expected to arrive. In this moment one has $V+V_{G}>$ $V_{B}$. The probability of the GDD to emit light when a MMW is absorbed can be modelled as [23]

$P_{G}=1-e^{-\alpha \eta_{G}}\left(1-p_{d G}\right)$. 
In (1), $\alpha$ is the mean photon number of the MMW, $\eta_{G}$ is the quantum efficiency of the GDD, that is, the probability of the MMW photon to be absorbed and at least one energetic electron to excite an atom that will suffer a radiative decay. At last, $p_{d G}$ is the probability of a photon to be emitted by the GDD when none MMW photon was absorbed. The light emitted by the GDD is thermal light since the same electron can excite more than one atom and, hence, more than one photon can be emitted per time. Since thermal light photon number distribution is given by $p_{n}=\mu_{t}^{n} /\left(1+\mu_{t}\right)^{1+n}$ the probability of the Si-SPD to flag photon detection is

$$
P_{T}=1-\left(1-p_{d}\right) /\left(1+\eta \mu_{t}\right)
$$

In (2) $\mu_{t}$ is the mean photon number of the thermal state produced by the GDD while $\eta$ and $p_{d}$ are, respectively, the quantum efficiency and the dark count probability of the SiSPD. Since the probability of the GDD to emit a light pulse with mean photon number $\mu_{t}$ is given by $P_{G}$ in eq. (1), $\mu_{t}$ depends on the values of $\eta_{G}$ and $p_{d G}$.

It is well known the GDD's ionization coefficient, $\sigma$, is given by:

$$
\sigma=p A \exp \left(-B p /\left(V_{T} / d\right)\right)
$$

where $A$ and $B$ are gas-specific and are derived from experiments, $p$ is the pressure, $d$ is the distance between the electrodes and $V_{T}$ is the voltage applied to the GDD. As a first approximation, one has $\mu_{t}=\alpha \sigma d \xi$ (considering the probability of an ionization equal to the probability of a radiative decay). Here, $\xi$ takes into account the coupling efficiency of light emitted by the GDD in the active region of the Si-APD. Therefore, the probability of $\mathrm{MMW} / \mathrm{THz}$ single-photon detection is

$$
P_{T}=1-\left(1-p_{d}\right) /\left[1+\eta \xi \alpha d p A \exp \left(-\frac{B p}{(V / d)}\right)\right]
$$

Equation (4) gives the photon detection probability as a function of the incident $\mathrm{MMW} / \mathrm{THz}$ radiation (mean photon number - $\alpha$ ), GDD's parameters $\left(A, B, p, d\right.$ and $\left.V_{T}\right)$, Si-SPD parameters $\left(\eta\right.$ and $p_{d}$ ) and the GDD/Si-SPD optical coupling ( $\xi$ ). For example, using $\alpha=0.1, p_{d}=10^{-5}, \eta=0.5, \xi=0.5, \sigma=$ $2300 \mathrm{~m}^{-1}$ and $d=3 \mathrm{~mm}$, one gets $P_{T}=0.1471$, per gate window of the Geiger mode.

\section{QUANTUM Key Distribution IN THE MiLlimeter-WAVE AND THz REGIONS USING GDD-SINGLE-PHOTON DETECTORS}

The GDD is sensitive to the polarization of the incident radiation $[21,22]$. The responsivity at $0 \mathrm{rad}$ rotation (the electric field of incident beam is parallel to the electric field between the electrodes of the GDD) is noticeably higher than the responsivity at $\pi / 2$ rotation (when the $\mathrm{DC}$ field and the $\mathrm{THz}$ electric field are orthogonal). This polarization dependence is relevant when one is measuring the variation of the electrical current however, it is not clear if it is relevant for the singlephoton detection operation, since in this case a strong or selfsustained cascade ionization is not required. Hence, the QKD protocol used will consider the MMW/THz always aligned with the electric field of the DC source.

The QKD protocol here considered is the B92 protocol [24]. The sender (Alice) will send $\mathrm{MMW} / \mathrm{THz}$ single-photons with polarization randomly chosen in the set $\{|0\rangle,|\pi / 4\rangle\}$. The horizontal polarization $|0\rangle$ is chosen with probability $p_{0}$ while the diagonal polarization $|\pi / 4\rangle$ is chosen with probability $p_{1}$. For each photon sent by Alice, the receiver (Bob) will choose with probability $1 / 2$ if the polarization of the incident photon will be or not rotated by $\pi / 4$. Bob's detection scheme is shown in Fig. 2.

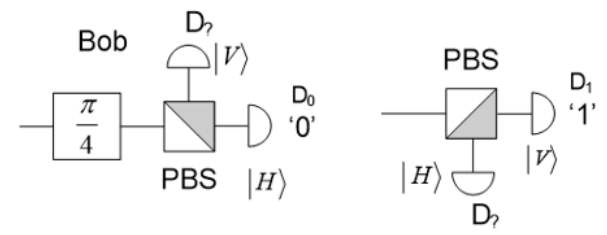

Fig. 2 - Bob's detection scheme. The PBS are polarizing beam splitters and $D_{0,1}$ are GDD-single-photon detectors.

According to the rules of B92 QKD protocol, when Alice sends a photon in the polarization state $|0\rangle$ and Bob applies the $\pi / 4$ rotation (left part of Fig. 2), with probability $1 / 2$ the photon will be guided to detector $\mathrm{D}_{0}$. If $\mathrm{D}_{0}$ fires, a bit ' 0 ' is recorded. On the other hand, when Alice sends a photon in the polarization state $|\pi / 4\rangle$ and Bob does not apply the $\pi / 4$ rotation (right part of Fig. 2), with probability $1 / 2$ the photon will be guided to detector $\mathrm{D}_{1}$. If $\mathrm{D}_{1}$ fires, a bit ' 1 ' is recorded. All the other situations are discarded (detections in $D_{\text {? }}$ ). Bob has to inform Alice the time slots he got valid detections. As usual, the detection of a spy (Eve) is inferred from the bit error rate (QBER). For a short channel (tens of meters) and negligible multipath dispersion, basically one has QBER $=P_{d G} P_{T}$.

In order to implement the B92 protocol, Alice and Bob uses a scheme similar to the one shown in [22], as shown in Fig. 3

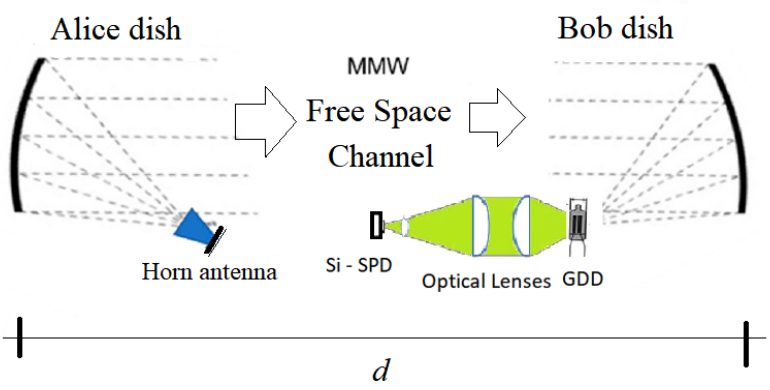

Fig. 3 - Scheme for realization of B92 QKD protocol with MMW.

The polarization modulated MMW beam is coupled out to free space using a horn antenna. The horn antenna was placed in the focal plane of an off-axis dish that collimates the MMW radiation and reflects it to a second off-axis dish that focuses the radiation on the GDD. Considering $\alpha_{0}$ the mean photon number of the MMW emitted by the horn antenna. The mean 
photon number of the MMW arriving at the GDD, $\alpha$, is given by (distances shorter than few tens of meters, atmospheric losses can be neglected).

$$
\alpha=\alpha_{0}\left[1-\exp \left(-D^{2} / 2\left(w_{0}^{2}+\frac{d w_{0} \lambda}{\pi}\right)\right)\right]=t \alpha_{0}
$$

In (5) $D$ is the diameter of the receiving element, $w_{0}$ is the beam waist leaving Alice, $d$ is the distance between Alice and Bob and $\lambda$ is the wavelength of the MMW. Thus, the raw key transmission rate is given by

$$
R=r-r\left(1-p_{d}\right) /\left[1+\eta \xi t \alpha d p A \exp \left(-\frac{B p}{(V / d)}\right)\right]
$$

In (6) $r$ is the rate the MMW pulses are sent to Bob. Equation (6) is the main result of our work.

\section{CONCLUSIONS}

The present work discusses the implementation of the B92 QKD protocol in the MMW and $(<10) \mathrm{THz}$ portion of the electromagnetic spectrum. Equation (6) is, on the best of our knowledge, the first transmission rate equation of a discrete variable QKD protocol operating in the $\mathrm{MMW} / \mathrm{THz}$ region. It can be fully implemented with current technology, Si-SPDs are commercially available and miniature neon lamps are very cheap. There are some practical challenges to be overcome, like the experimental determination of quantum efficiency and dark count probability of the GDD-Si-SPD, the determination of the GDD voltage that will optimize the NEP of the detector and the maximal transmission rate, since secondary electrons can be emitted by the GDD when the ions reach the cathode and those electrons can start a new photon emission. This effect is similar to afterpulsing in APDs and should be avoided. Since the implementation of the QKD protocol here proposed is simple, it can be easily built and its performance can be checked.

\section{REFERENCES}

[1] K. Inoue, Differential phase-shift quantum key distribution systems, IEEE Sel. Top. in Quant. Elec., 21, 3, 6600207, 2015.

[2] C. H. Bennett, Quantum cryptography using any two non-orthogonal states, Phys. Rev. Lett,68, 3121, 1992.

[3] K. Tamaki e N. Lütkenhaus, Unconditional security of the Bennet 1992 protocol quantum key-distribution over lossy and noisy channel. http://xxx.lanl.gov - quant-ph/0308048, 2003.

[4] F. A. Mendonça, D. B. de Brito, J. B. R. Silva, G. A. P. Thé, R. V. Ramos, Experimental implementation of B92 quantum key distribution protocol, 50, 1, 236-241, 2008.

[5] F. Laudenbach, C. Pacher, C.-H. F. Fung, A. Poppe, M. Peev, B. Schrenk, M. Hentschel, P. Walther, H. Hübel, Continuous-Variable Quantum Key Distribution with Gaussian Modulation - The Theory of Practical Implementations, Adv. Quantum Technol.,1, 1800011/1-37, 2018.

[6] Y. Jian, E. Wu, G. Wu, and H. Zeng, "Optically Self-Balanced InGaAsInP AvalanchePhotodiode for Infrared Single-Photon Detection", IEEE Photo. Tech. Lett. 22, 3, 173-175, 2010.
[7] Y. Liang, Q. Fei, Z. Liu, K. Huang, H. Zeng, "Low-noise InGaAs/InP single-photon detector with widely tunable repetition rates", Photonics Research, 7, 3, A1-A6, 2019.

[8] N. Namekata, S. Sasamori, and S. Inoue, "800 MHz Single-photon detection at 1550-nm using an InGaAs/InP avalanche photodiode operated with a sine wave gating", Opt. Expresss, 14, 21, 10043-1049, 2006.

[9] Z. L. Yuan, A. R. Dixon, J. F. Dynes, A. W. Sharpe, and A. J. Shields, "Gigahertz quantum key distribution with InGaAs avalanche photodiodes", Appl. Phys. Lett. 92, 20, 201104, 2008.

[10] G. C. Silva, J. S. de Andrade, R. L. C. Damasceno, J. B. R. Silva, R. V. Ramos, Remotely Gated InGaAs Single-Photon Detector at $1550 \mathrm{~nm}$, IEEE Photonics Technology Letters, 32, 2, 129-131, 2020.

[11] X. You at al, Towards $6 \mathrm{G}$ wireless communication networks: vision, enabling technologies, and new paradigm shifts, Sci. China Info. Sciences 64, 110301, 2021. doi: 10.1007/s11432-020-2955-6

[12] C. Weedbrook, S. Pirandola, S. Lloyd, and T. C. Ralph, Quantum cryptography approaching the classical limit, Phys. Rev. Lett. 105, 110501/1-4, 2010.

[13] C. Weedbrook, S. Pirandola, and T. C. Ralph, Continuous-variable quantum key distribution using thermal states, Phys. Rev. A 86, 022318/1-12, 2012.

[14] Y. He, Y. Mao, D. Huang, Q. Liao, Y. Guo, Indoor channel modeling for continuous variable quantum key distribution in the terahertz band, Opt. Express, 28, 22, 32386-32402, 2020.

[15] N. S. Kopeika, Glow discharge detection of long wavelength electromagnetic radiation: Cascade ionization process internal signal gain and temporal and spectral response properties, IEEE Transactions on Plasma Science, PS-6, No. 2 139-157, 1978.

[16] L. Kahana, D. Rozban, M. Gihasi, A. Abramovich, Y. Yitzhaky and N. Kopeika, Inexpensive Millimeter-Wave Communication Channel Using Glow Discharge Detector and Satellite Dish Antenna, Electronics, 9, 677, 1-8, 2020; doi:10.3390/electronics9040677

[17] A. Abramovich, N. S. Kopeika, D. Rozban, E. Farber, Terahertz detection mechanism of inexpensive sensitive glow discharge detector. J. Appl. Phys. 103, 093306, 2008.

[18] L. Hou, W. Shi, Fast terahertz continuous-wave detector based on weakly ionized plasma. IEEE Electron Device Lett. 33, 1583-1585, 2012.

[19] A. Aharon, D. Rozban, N. S. Kopeika, and A. Abramovich, Heterodyne detection at $300 \mathrm{GHz}$ using neon indicator lamp glow discharge detector, Applied Optics, 52, 17, 4077-4082, 2013.

[20] A. Aharon, D. Rozban, A. Klein, A. Abramovich, Y. Yitzhaky, N. S. Kopeika, Detection and upconversion of three-dimensional MMW/THz images to the visible, Photon. Res. / Vol. 4, No. 6, 306-312, 2016.

[21] A. Abramovich, N. S. Kopeika, and D. Rozban, THz Polarization Effects on Detection Responsivity of Glow Discharge Detectors (GDDs), IEEE Sensors Journal, 9, 10, 1181-1184 2009.

[22] T. Takan, N. Alasgarzade, I. U. Uzun-Kaymak, A. B. Sahin, H. Altan, Detection of far-infrared radiation using glow discharge detectors, Opt. Quant. Electron., 48, 292/1-10, 2016. DOI 10.1007/s11082-016-0569-Z

[23] V. F. Guedes, F. A. Mendonca, J. B. R. Silva, R. V. Ramos, A Proposal for Single-Photon Detection in Millimeter-Wave and THz Regions, submitted to IMOC 2021, Fortaleza-Brazil, 2021.

[24] G. S. Castro, R. V. Ramos, Detecting Eavesdropping Activities in BB84 Quantum Key Distribution Protocol Using the Disentropy of the Autocorrelation, ResearchGate, 2020.

[25] R. V. Ramos, Estimation of the Randomness of Continuous and Discrete Signals Using the Disentropy of the Autocorrelation, ResearchGate, 2020.

This work was supported by the Brazilian agencies CNPq via Grant no. 307184/2018-8 and CAPES via no. Grant 001. Also, this work was performed as part of the Brazilian National Institute of Science and Technology for Quantum Information. The authors are with Teleinformatic Engineering Department, Federal University of Ceara, Fortaleza, Brazil. (emails: vitorfergue@gmail.com, fabioalencar@ifce.edu.br, joaobrs@ufc.br, rubens.ramos@ufc.br). 